\title{
Guía para el autor de estudios de caso: cómo investigar, escribir, enseñar y evaluar
}

\author{
Case study author's guide: how to research, write, teach and evaluate
}

\author{
Francisco Arias ${ }^{1} y$ Orlando Betancur ${ }^{2}$
}

Revista

\section{Educación y Sociedad}

Citar como: Arias, F. y Betancur, O. (2020). Guía para el autor de estudios de caso: cómo investigar, escribir, enseñar y evaluar. Revista Educación y Sociedad, 01(02), 31-43. doi: $10.53940 /$ reys.v1i2.57

Artículo recibido: 12-11-2020 Artículo aprobado: 20-12-2020 Arbitrado por pares

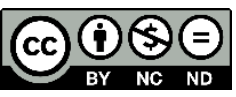

\section{ACEES}

\section{Resumen}

La metodología de estudio de caso se constituye como una alternativa pedagógica para la investigación y la enseñanza en el campo empresarial. Este trabajo propone una ruta para investigar, escribir, enseñar y evaluar estudios de caso en programas académicos de corte empresarial. Se realiza una aproximación mediante la técnica de análisis documental y el uso de matrices categoriales para sintetizar las pautas metodológicas. Como resultado, se tiene una compilación de los principales aportes que se tienen en el proceso de implementación de estudios de caso.

Palabras clave: caso, estudio, empresa, investigación, evaluación

\section{Abstract}

The case study methodology is constituted as a pedagogical alternative for research and teaching in the business field. This paper proposes a route to research, write, teach and evaluate case studies in entrepreneurial academic programs. An approach is made through the technique of documentary analysis and the use of categorical matrices to synthesize the methodological guidelines. As a result, we have a compilation of the main contributions to the process of implementing case studies.

Key words: case, study, company, research, evaluation 


\section{Introducción}

Una de las metodologías de pedagogía activa que viene ganando interés entre los académicos es el estudio de caso, el cual ha demostrado ser una herramienta educativa que permite contrastar la práctica con la teoría, permitiendo una mejor comprensión de los conceptos y el abordaje de aspectos complejos en ciencias empresariales (Yin, 2013); también ha sido una metodología valiosa que ha permitido investigar aspectos que habían permanecido de manera tácita como lo ocurrido en múltiples organizaciones y que mediante el desarrollo de un proceso de sistematización e incorporación del método científico ha posibilitado que ese conocimiento hoy en día sea explicito (Castro Monge, 2010).

Una de las razones de su éxito en el mundo académico es la posibilidad de integrar conceptos para la solución a problemas de situaciones reales o simuladas en un contexto de aprendizaje experiencial, en vez de continuar con los modelos tradicionales educativos que buscan tener un conocimiento parcelado , pero que en ocasiones son difíciles de integrar y comunicarse entre sí, dejando a los estudiantes con la duda de cómo se aplica una u otra asignatura o conocimiento específico en el mundo real (Ridder, 2017).

Son varios los investigadores que en el campo de las ciencias empresariales han documentado los beneficios sobre una correcta enseñanza a partir de estudios de caso en la formación de competencias y habilidades directivas (Culpin y Scott, 2012; Shivakumar, 2012; Sridevi, 2012); pues se convierte en laboratorio dinámico de aprendizaje donde el experimento, si es conducido de forma adecuada, puede no solo mejorar las habilidades directivas de los sujetos, sino garantizar un aprendizaje correcto, lo cual es posible si se traza una ruta pedagógica desde la programación de los cursos del plan de carrera y se verifica en colectivos docentes el alcance de los mismos (Keevy, 2016; Mahdi, Nassar, y Almuslamani, 2020).

Una de las críticas - injustas, por cierto - que se hacen al estudio de caso, es que muchos deben de ser comprarlos a ciertas universidades a precios elevados; lo que dificulta el acceso a facultades de negocios con menos capacidades económicas como lo son usualmente las instituciones universitarias en países emergentes. Lo cual generaría inequidad ya que el conocimiento estaría disponible al que pueda solventarlo económicamente.

De otro lado, al abordar el caso de estudio es importante no limitarse al contexto de las preguntas que usualmente están al final de los documentos y que en ocasiones podrían rayar dentro del campo de la subjetividad, por el hecho de quien conduce el caso puede desconocer parcial o totalmente hechos o la solución del mismo (Diefenbach, 2009; Shugan, 2006). Para superar esta brecha, es importante la existencia de materiales complementarios y notas de enseñanza, para que se pueda contar con el soporte adecuado, y, asimismo, preparar los elementos necesarios para abordar la praxis con un nivel adecuado de profundidad. Todo esto, con miras a conservar el rigor científico y, al mismo tiempo, salvaguardar la conquista de aprendizajes significativos.

Otro reto que tiene la metodología son los ataque que se reciben por parte de escépticos que argumentan el hecho de que, en ocasiones, los profesores escogen algún material en particular, se hace la discusión y la solución, y en este preciso momento, el estudiante puede salir con la falsa sensación que aprendió (Shugan, 2006); situación que plantea la necesidad de dar garantías de aprendizaje y evidencias que bien se podrían apreciar a través de rúbricas.

También, existen diferentes tipologías de estudios de caso que nos llevan comprender de que existe un método único para hacer investigación, ya que el objeto, el contexto y la intencionalidad puede hacer que se acuda a una técnica en específico o a una combinación de las mismas. Asimismo, y en esta diversidad, tampoco existe una forma única de enseñar y mucho menos de evaluar (Rave y Franco, 2011). 
En este contexto, la presente investigación pretende compilar el desarrollo alcanzado en torno a esta metodología y desde la diversidad de enfoques con respecto escribir, enseñar y evaluar; con el propósito de superar la perspectiva fragmentada que por general caracteriza a este campo. También, merece mencionarse que estos despliegues investigativos se sustentan en los hallazgos previos y reflexiones gestadas en el seno de la Red Internacional de Investigación en Gestión del Conocimiento Empresarial (RED GCE).

\section{Metodología}

El presente trabajo se enmarca en un proceso cualitativo de análisis documental (Barbosa, Barbosa, y Rodríguez, 2013) y el desarrollo de matrices categoriales (Corbetta, Fraile, y Fraile, 2007) con el propósito de identificar pautas relacionadas en la literatura de estudios de caso. Esto, con focos de atención referidos a las pautas requeridas para escribir un estudio de caso, a la manera de enseñar bajo esta metodología y al cómo evaluar formativamente - con el soporte de rúbricas - las soluciones planteadas por los estudiantes en la sala de clases (Arias, Garcés, Betancourt, Sepúlveda, y Arboleda, 2020).

Para desarrollar lo anterior; se diseñó una ecuación de búsqueda (ver ecuación 1), según los parámetros de exploración propuestos por Codina, (2017), utilizando además operadores booleanos para refinar los criterios de búsqueda (LI. Codina, 2018; Gallardo, 2013).

$$
\mathrm{F}(\mathrm{x})=\text { ("Case study" AND (write AND teach OR evaluate)) }
$$

La anterior expresión arrojó un resultado amplio de 133 documentos en bases de datos de reconocida trayectoria como Scopus, Web of Sciences (WoS) y Google académico, los cuales se revisaron de forma sistemática en cuanto al título y resumen para generar la coincidencias de búsqueda requerida, lo cual se sumó a libros de reconocidos autores en el área para generar una base de 383 documentos gestionados con el Software Mendeley (Codina y Morales, 2019), con los cuales se construyó el presente documento.

\section{Resultados y discusión}

La revisión documental muestra un gran interés por la temática de estudio de caso, encontrándose alrededor de 3,9 millones de documentos (ver figura 1), los cuales abordan la metodología desde diferentes perspectivas tales como la comprensión de teorías, la interacción de saberes fragmentados a nivel académico, el análisis de procesos de cambio, estudios organizacionales, aspectos culturales y laborales, revisión de fenómenos propios de la empresa. Por otro lado, existe otro enfoque centra do en la metodología y su efectividad, sobre el cómo mejorar los procesos investigativos, hacer comparaciones de resultados y conceptuales, así como el motivar la interacción de docentes con estudiantes para mejorar el desempeño, entrenar a los nuevos profesionales y permitir el desarrollo de habilidades y competencias directivas en los sujetos (Culpin, Eichenberg, Hayward, y Abraham, 2014; Keevy, 2016). 


\section{Documents by year}

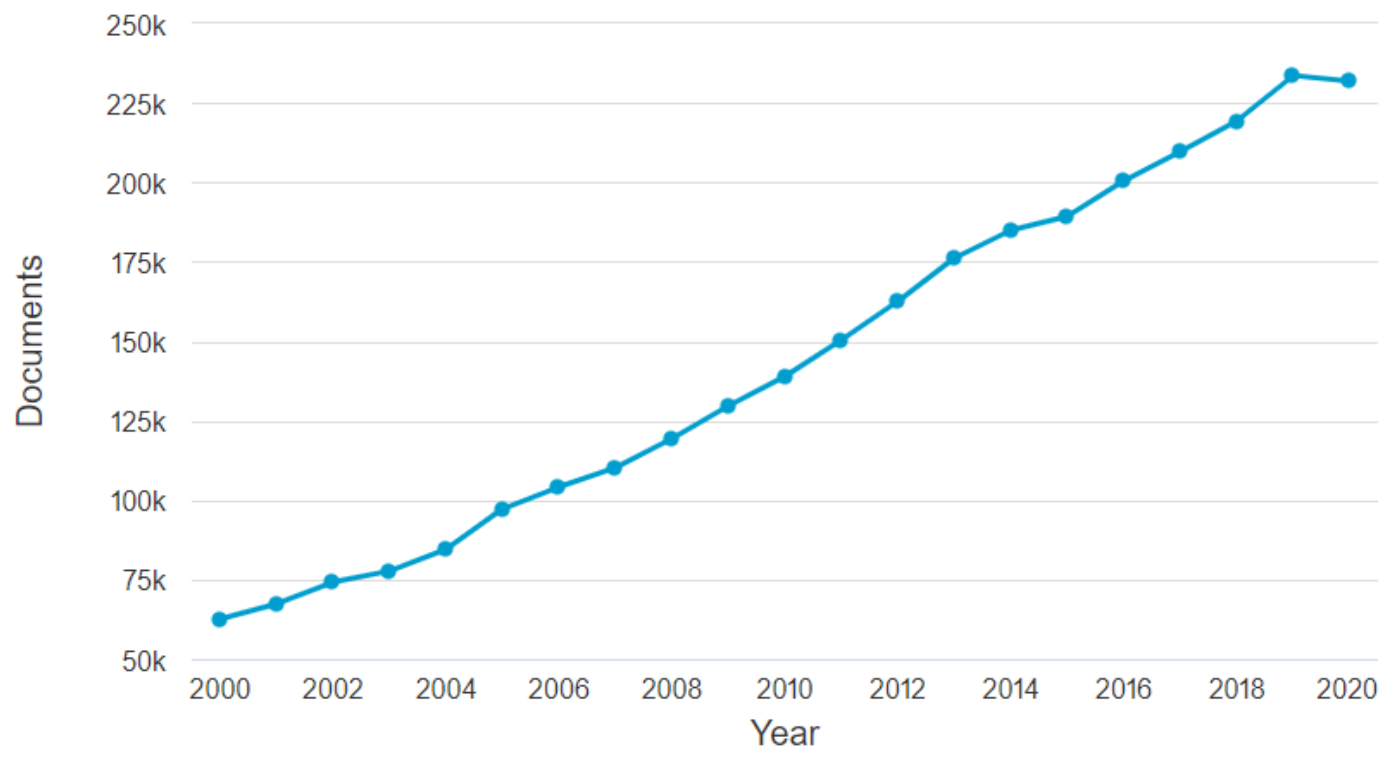

Figura 1. Evolución de publicaciones en estudios de caso (2000-2020)

Fuente: Scopus

Existen autores que han propuesto diversos métodos y formas para la construcción de estudios de caso de enseñanza, siendo el modelo Harvard el de mayor acogida por los cuerpos académicos a nivel internacional; donde frecuentemente se ilustran las prácticas y las estrategias que han llevado a las organizaciones a obtener el éxito empresarial. Sin embargo, existen críticas puesto que las universidades consideradas de elite, tienden a centrarse en las grandes empresas, descuidando en ocasiones las realidades locales en las cuales están inmersas muchas de las economías emergentes y dónde la representatividad de las PYMES puede llegar a constituir el 90\% o más del tejido empresarial.

\section{Recomendaciones de como investigar y escribir un caso de enseñanza}

Existen diversos autores que han propuesto rutas y pautas para investigar basados en la metodología de estudios de caso, promovido por autores como Robert Stake (Stake, 1995, 2004) y Robert Yin (Yin, 2012, 2013), a los cuales se han sumado otros estudiosos del tema que han generado importantes (Arias et al., 2020; Boblin, Ireland, Kirkpatrick, y Robertson, 2013; Camacho Gómez, 2011; Culpin et al., 2014; Dooley, 2002; Flyvbjerg et al., 2006; Martínez Carazo, 2006; Morgan, Pullon, Macdonald, McKinlay, y Gray, 2016; Rave y Franco, 2011; Yacuzzi, 2005).

Al realizar la revisión documental, se pueden establecer un conjunto de 22 etapas que se deben surtir para llevar un adecuado proceso investigativo (ver Tabla 1). 
Tabla 1

Elementos para investigar con la metodología de caso de estudio

\begin{tabular}{cl}
\hline $\mathrm{N}^{\circ}$ & \\
\hline 1 & Estar interesado, conocer y/o estudiar el sector sobre el cual se pretende escribir el caso \\
2 & Seleccionar una lista de posibles empresas y seleccionar la más viable según los criterios \\
3 & An investigación \\
4 & Seleccionar el tema central del caso \\
5 & Formular el problema de investigación \\
6 & Proponer la hipótesis o el conjunto de ellas \\
7 & Revisar si existen temas que se conectan con el foco del caso \\
8 & Explicar a la empresa que se pretende con el caso y que insumos se requieren para \\
& construirlo \\
9 & Definir a la empresa que garantías tiene y que ganara con la realización del caso \\
10 & Solicitar permisos y accesos a la información \\
11 & Establecer con la empresa asuntos de confidencialidad de información. \\
12 & Determinar qué se puede incluir en el caso, qué se autoriza y qué no (para que el \\
13 & investigador defina si es pertinente o no continuar el ejercicio investigativo) \\
14 & Definir los recursos necesarios (dinero, personal, etc.) \\
15 & Realizar trabajo de campo \\
16 & Recopilar información utilizando fuentes primarias y secundarias \\
17 & Procesar la información cualitativa y cuantitativa \\
18 & Triangular la información \\
19 & Articular con la literatura académica \\
20 & Redactar el caso y las preguntas orientadoras \\
21 & Desarrollar materiales complementarios y/o sugerirlo \\
22 & Realizar la nota de enseñanza y/o la guía de clase \\
\hline
\end{tabular}

Fuente: (Arias et al., 2020).

Después del desarrollo investigativo propuesto en la Tabla 1, viene una de las etapas más importantes que es la escritura del material académico; el cual puede ser escrito con la estructura propia de un artículo científico, capítulo de libro o incluso libro (Eisenhardt, 1991; George, 2019; Siggelkow, 2007; Welch, Piekkari, Plakoyiannaki, y Paavilainen-Mäntymäki, 2011; Yin, 2013). Incluso, hay quienes documentan sus hallazgos con una narrativa propia y desde una perspectiva literaria, creando historias y cuentos a partir de sus hallazgos (Camacho Gómez, 2011; Ogliastri, 1998).

En la redacción del caso, es recomendable adoptar una estructura simple para que sea entendible por el público de pregrado y posgrado sin dejar de lado el rigor y la solvencia teórica. Así, y en virtud a esto, el autor debe buscar una construcción efectiva, procurando la existencia de elementos propios que interactúen con otras áreas del conocimiento a nivel disciplinar y transdisciplinar (Crosbie, 2005; Harland, 2014; Sridevi, 2012).

La estructura propuesta para la construcción de un caso de estudio (ver tabla 2), presenta la estructura propia que debería tener un caso de enseñanza: 
Tabla 2

Estructura propuesta del caso de estudio

\begin{tabular}{cl}
\hline$N^{\circ}$ & \multicolumn{1}{c}{ Elementos } \\
\hline 1 & Resumen \\
2 & Temas que cubre el caso \\
3 & Introducción \\
4 & Pregunta de investigación \\
5 & Metodología \\
6 & Reseña histórica y/o marco teórico \\
7 & Desarrollo del foco y/o temática \\
8 & Análisis sectorial \\
9 & Prospectiva y/o proyección \\
10 & Conclusiones \\
11 & Preguntas para el análisis \\
12 & Lecturas complementarias sugeridas (textos, páginas web) \\
13 & Bibliografía \\
14 & Nota de enseñanza \\
15 & Material complementario y anexos \\
\hline Fuente: (Arias et al., 2020).
\end{tabular}

La nota de enseñanza es un material pedagógico dirigido especialmente al docente que va a llevar la sesión de estudio; el cual debe garantizar unos estándares mínimos de calidad en cuanto al aprendizaje (Naumes y Naumes, 2015) y la ética sobre lo que se aprende (Rendtorff, 2015). Del mismo modo, se debe procurar que el proceso de enseñanza sea el adecuado y, por consiguiente, que los estudiantes estén aprendiendo correctamente (Wai y Seng, 2013).

Una de las características que favorecería el aprendizaje en las circunstancias en las que se dio el desarrollo del estudio de caso, es la nota de enseñanza, la cual se constituye como un material pedagógico orientador para guiar una solución de manera adecuada las cuales podrían tener los siguientes elementos sugeridos (ver Tabla 3). 
Tabla 3

Elementos que debería tener una nota de enseñanza

\begin{tabular}{cl}
\hline$N^{\circ}$ & \multicolumn{1}{c}{ Elementos } \\
\hline 1 & Tipo de público para ser aplicado \\
2 & Localización geográfica y temporal de la empresa y el caso \\
3 & $\begin{array}{l}\text { Clasificación del caso (Extrínseco, intrínseco, exploratorio, } \\
\text { descriptivo, ilustrativo, explicativo, etc.) }\end{array}$ \\
4 & Síntesis del caso \\
5 & Temas que cubre el caso \\
6 & Habilidades y/o competencias a desarrollar \\
7 & Aprendizajes previos que debiesen tener los estudiantes \\
8 & Materiales complementarios \\
9 & Recomendaciones a los docentes sobre aprendizajes transversales \\
10 & y complementarios con otras disciplinas \\
11 & Estrategias para mejorar el proceso de enseñanza aprendizaje \\
12 & Experiencias previas en la utilización y aplicación de los estudios \\
& de caso \\
13 & Relatar que ocurrió realmente \\
14 & Rubricas de evaluación del caso \\
\hline
\end{tabular}

Fuente: (Arias et al., 2020).

\section{Recomendaciones sobre cómo enseñar a partir de estudios de caso}

No existe una manera única de enseñar con estudios de caso ya que cada docente que enseñan imprime sus propios estilos o formas didácticas. Sin embargo, es importante seguir unas pautas que permitan, a partir de las diferencias, cristalizar despliegues académicos articulados armónicamente para que se cumplan los propósitos formativos del estudio de caso. El propósito es buscar una interacción entre el sujeto, el equipo, el contexto, la teoría y la práctica que conduciría más que a una solución propiamente en sí a un aprendizaje significativo, formativo y experiencial.

Existen técnicas que va desde el desarrollo tradicional del estudio de casos donde el maestro entrega el material para ser leído y responder unas preguntas orientadoras en un contexto de clase. Otros hacen uso de representaciones teatrales y escenarios simulados. Asimismo, un grupo importante de educadores recurren a medios audiovisuales u optan por desarrollar casos en vivo directamente en los escenarios donde ocurren los hechos, usualmente con personajes de la vida real tales como gerentes y personal clave; hasta el uso de material interactivo que contempla cursos de acción diferentes dependiendo de las elecciones que van dando los sujetos al desarrollo propio del caso.

De esta manera, e independiente de la estrategia utilizada, existe evidencia empírica de la interacción de los estudiantes con el método de caso en el cual la teoría cobra sentido, motiva la creatividad y el deseo por conocer aspectos por cuenta propia a través de la indagación complementaria.

La preparación del curso debe integrar la teoría y los casos seleccionados por el docente deben armonizar con los propósitos formativos para que estos no se vean sueltos y lleven al estudiante en una secuencia lógica de aprendizaje (Tecnológico de Monterrey, 2010); sin embargo, es esencial que los 
ejercicios de casuística no sean esfuerzos aislados por parte de los docentes que quieren preparar mejor a sus estudiantes. Por el contrario, debe ser un esfuerzo coordinado por parte del programa, la facultad e incluso la institución educativa para que se puedan lograr los fines deseados.

La preparación de casos a medida para articular el avance teórico del curso que se lleva o la integración de asignaturas (si el desarrollo educativo se da de esta forma) requiere una adecuada preparación del personal docente con la metodología, la investigación, el desarrollo de la escritura y la aplicación en clase de los casos de estudio. Por ello, se deben tener en cuenta las siguientes recomendaciones (ver Tabla 4).

Tabla 4

Puntos a revisar para la enseñanza a partir de casos empresariales

$\mathrm{N}^{\circ} \quad$ Elementos

Preparar el sylabus o también denominado micro currículo en el cual se exprese

1 el tipo de casos, número y sesiones de trabajo dentro del marco de la asignatura

2 Revisar el número, calidad y objetivos de los casos seleccionados

3 Analizar que conceptos teóricos que se relacionan con los diferentes momentos del curso y con los casos planteados

4 Definir la estrategia evaluativa

5 Inducir al estudiante en que consiste la metodología y que se espera de su trabajo y aportes tanto a nivel individual como de equipo

6 Asegurarse de solicitar los entregables y preparaciones previas a los estudiantes para asumir la metodología

7 Hacer un seguimiento minucioso del desarrollo del caso y los objetivos de aprendizaje en las diferentes sesiones donde se trabaje

8 Propiciar el trabajo colaborativo y la discusión grupal argumentada

9 Detonar discusiones problémicas mediante preguntas orientadoras a los estudiantes

10 Relacionar conocimientos de otras áreas y motivar la indagación a profundidad por parte del estudiante

11 Propiciar la creatividad y la innovación

12 Evaluar de manera grupal las posibles decisiones y las implicaciones de las soluciones generadas en clase

13 Incorporar en la evaluación otros colegas para que puedan realizar aportes significativos desde diferentes áreas del conocimiento

14 Realizar retroalimentación

Fuente: (Arias et al., 2020).

Respecto a la ruta de aprendizaje, el profesor Enrique Ogliastri propuso el siguiente esquema en el cual se debe preparar el caso abordando los hechos y en donde posteriormente debe generar un análisis minucioso apoyándose en la teoría, prácticas y experiencias propias del docente y los estudiantes. Esto, con el fin de contemplar todos los hechos posibles, focalizarse en el problema y comprenderlo en profundidad para establecer posibles salidas, soluciones o abanico de decisiones pertinentes al caso debidamente contextualizado (ver figura 2): 


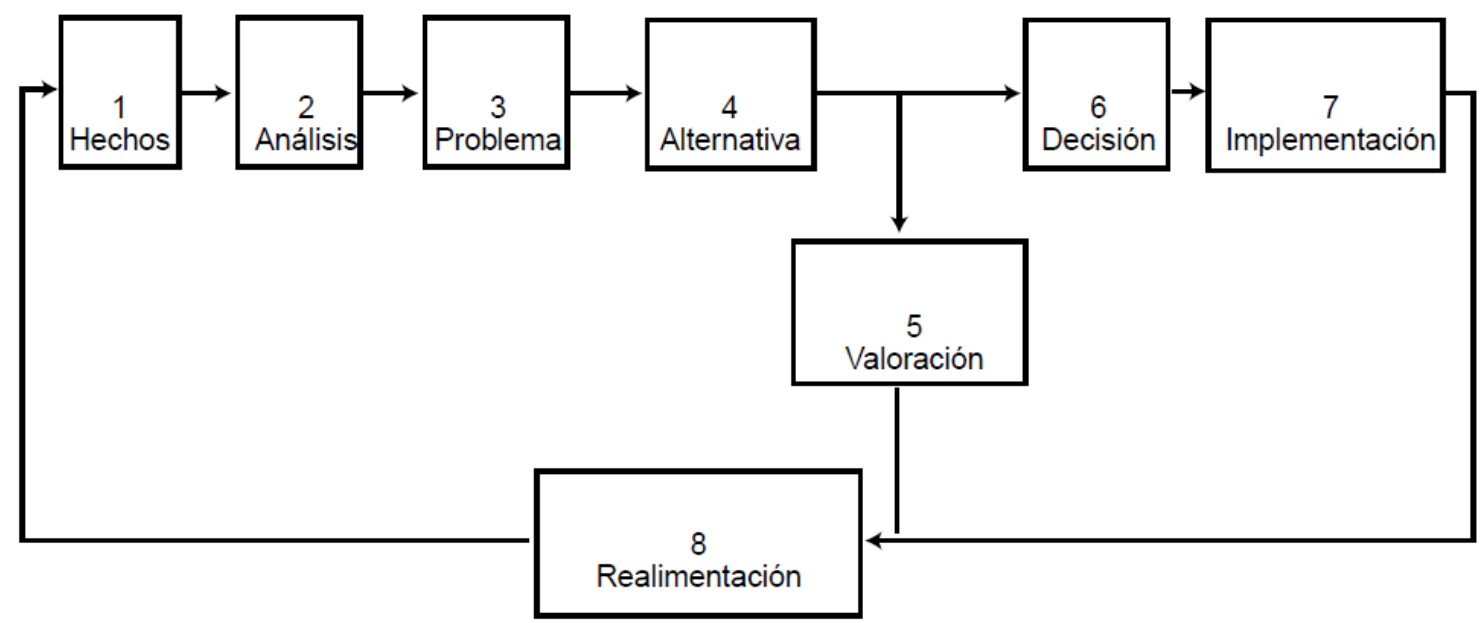

Figura 2. Proceso de enseñanza a partir del método de estudio de caso Fuente: Tomado de Ogliastri (1998, p. 16).

\section{Recomendaciones de evaluación estudios de caso: la rúbrica de evaluación}

Uno de los aspectos que aporta claridad al proceso de enseñanza-aprendizaje es el desarrollo de una rúbrica de evaluación por parte del docente o el autor del caso; sugiriendo un enfoque orientado hacia temas específicos que deben complementarse con la discusión académica entre pares y con la participación activa por parte de los responsables del curso.

Frente a lo anterior, Gordillo y Rodríguez (2010), mencionan que:

La rúbrica es un instrumento de evaluación basado en una escala cuantitativa y/o cualitativa asociada a unos criterios preestablecidos que miden las acciones del alumnado sobre los aspectos de la tarea o actividad que serán evaluados. Básicamente, existen dos grupos: las holísticas, que tratan de evaluar el aprendizaje o competencia desde una visión más global, y las analíticas, que se centran en algún área concreta de aprendizaje. Además, nos permite diseñarla para tareas amplias o específica (p.142).

Por lo tanto, el desarrollo de una rúbrica para estudios de caso, debe ser consecuencia del proceso de estructuración del conocimiento que permitió su escritura y el cual debe contar con elementos mínimos (ver Tabla 5), para evaluar el aprendizaje a nivel individual o colectivo de una forma integral (Chan, Inoue, y Taylor, 2015; Clary, Brzuszek, y Fulford, 2011; Llanes y Massot, 2013; Maggin, Briesch, Chafouleas, Ferguson, y Clark, 2014; Noblitt, Vance, y Smith, 2010; Riddle, Smith, y Frankforter, 2016). 
Tabla 5

Propuesta de rúbrica para evaluar la solución de los casos de enseñanza

\begin{tabular}{|c|c|c|c|c|c|c|c|}
\hline Perspectiva & Ítem & Criterio & Excelente & Bueno & Regular & Deficiente & Insuficiente \\
\hline \multirow{7}{*}{ 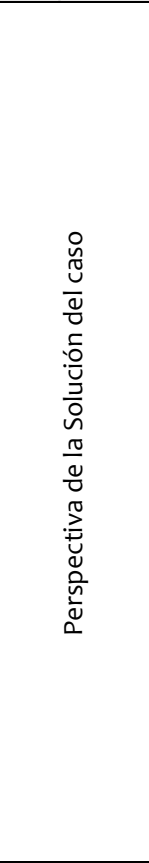 } & $\begin{array}{l}\text { Presentación del } \\
\text { caso }\end{array}$ & $\begin{array}{l}\text { Coherente y } \\
\text { adecuadamente } \\
\text { documentado }\end{array}$ & & & & & \\
\hline & Lectura detenida & $\begin{array}{l}\text { Se realiza una adecuada } \\
\text { compresión de lectura y del } \\
\text { contexto }\end{array}$ & & & & & \\
\hline & $\begin{array}{l}\text { Identificación de } \\
\text { lo que se pide }\end{array}$ & $\begin{array}{c}\text { El o los estudiantes } \\
\text { comprenden el alcance de } \\
\text { las preguntas orientadoras } \\
\text { del caso y/o el docente? }\end{array}$ & & & & & \\
\hline & $\begin{array}{l}\text { El caso tiene } \\
\text { solución }\end{array}$ & $\begin{array}{c}\text { Pasar a las etapas de } \\
\text { resolución }\end{array}$ & & & & & \\
\hline & $\begin{array}{l}\text { El caso no tiene } \\
\text { solución }\end{array}$ & $\begin{array}{l}\text { Argumentar desde una } \\
\text { posición teórica porque no } \\
\text { se puede llegar a una } \\
\text { solución }\end{array}$ & & & & & \\
\hline & Extras & $\begin{array}{c}\text { ¡Él o los estudiantes } \\
\text { entregan elementos } \\
\text { adicionales, perspectivas } \\
\text { no contempladas o } \\
\text { aspectos poco evidentes } \\
\text { del caso abordado? }\end{array}$ & & & & & \\
\hline & $\begin{array}{l}\text { Tiempos de } \\
\text { resolución del } \\
\text { caso }\end{array}$ & $\begin{array}{l}\text { El o los estudiantes } \\
\text { entregaron a tiempo }\end{array}$ & & & & & \\
\hline \multirow{4}{*}{ 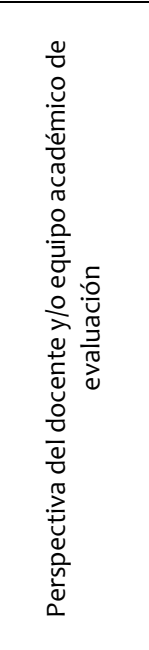 } & $\begin{array}{l}\text { Análisis de } \\
\text { alternativas } \\
\text { diferentes a las } \\
\text { soluciones } \\
\text { tradicionales } \\
\end{array}$ & $\begin{array}{l}\text { ¿El o los estudiantes } \\
\text { proponen alternativas } \\
\text { diferentes, creativas y } \\
\text { posibles al desafío } \\
\text { planteado? }\end{array}$ & & & & & \\
\hline & $\begin{array}{l}\text { Competencias } \\
\text { y/o habilidades } \\
\text { evidenciadas por } \\
\text { el docente }\end{array}$ & $\begin{array}{l}\text { Competencia (s) y/o } \\
\text { habilidad (es) que el } \\
\text { docente pretenda medir }\end{array}$ & & & & & \\
\hline & Argumentación & $\begin{array}{c}\text { El o los estudiantes } \\
\text { argumentan } \\
\text { adecuadamente los } \\
\text { conceptos o soluciones } \\
\text { que proponen } \\
\end{array}$ & & & & & \\
\hline & Uso de teorías & $\begin{array}{l}\text { ¿Los estudiantes } \\
\text { argumentan desde } \\
\text { posiciones teóricas la } \\
\text { solución o posibles } \\
\text { soluciones del caso? }\end{array}$ & & & & & \\
\hline \multirow{6}{*}{ 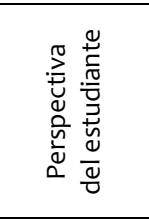 } & \multirow{6}{*}{$\begin{array}{l}\text { Autoevaluación } \\
\text { por parte del } \\
\text { estudiante }\end{array}$} & Participación & & & & & \\
\hline & & Responsabilidad & & & & & \\
\hline & & Dinámica de trabajo & & & & & \\
\hline & & Actitud de trabajo & & & & & \\
\hline & & Trabajo en equipo & & & & & \\
\hline & & Responsabilidad & & & & & \\
\hline \multirow{4}{*}{ 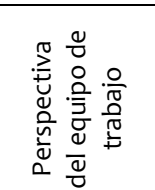 } & \multirow{4}{*}{$\begin{array}{l}\text { Evaluación del } \\
\text { equipo de } \\
\text { trabajo a cada } \\
\text { uno de sus } \\
\text { miembros }\end{array}$} & Dinámica de trabajo & & & & & \\
\hline & & Actitud de trabajo & & & & & \\
\hline & & Trabajo en equipo & & & & & \\
\hline & & Responsabilidad & & & & & \\
\hline
\end{tabular}

Si bien lo anterior es una propuesta de rubrica, cada maestro puede incorporar elementos no contemplados aquí con el propósito de mejorar los instrumentos de evaluación del desempeño estudiantil. Además, esta rúbrica permitiría identificar tanto fortalezas como debilidades con miras a la mejora constante. 


\section{Conclusiones}

Este trabajo reúne una serie de pautas distribuidas en la literatura de estudios de caso y ha procurado abordar en un solo documento algunos criterios que deben ser tenidos en cuenta para investigar, escribir, enseñar y evaluar. Asimismo, es importante anotar que los estudios de caso es un área que está en una constante dinámica de cambio, y por ello, es importante continuar actualizando los elementos que acompañan el proceso de enseñanza aprendizaje en función de los cambios tecnológicos y los hallazgos alrededor del mundo en este campo de conocimiento.

\section{Referencias}

Arias, F., Garcés, L., Betancourt, J., Sepúlveda, J., y Arboleda, C. (2020). Los estudios de caso como práctica pedagógica para formación en competencias empresariales. En Diagnóstico, estrategias e innovación empresarial: enfoques multidisciplinarios (pp. 30-49). Medellín: Sello Editorial Americana.

Barbosa, J.W., Barbosa, J.C., y Rodríguez, M. (2013). Revisión y análisis documental para estado del arte: una propuesta metodológica desde el contexto de la sistematización de experiencias educativas. Investigación Bibliotecológica, 27(61), 83-105. doi: 10.1016/S0187-358X(13)72555-3

Boblin, S. L., Ireland, S., Kirkpatrick, H., y Robertson, K. (2013). Using Stake's qualitative case study approach to explore implementation of evidence-based practice. Qualitative Health Research, 23(9), 1267-1275. Recuperado de http://www.ncbi.nlm.nih.gov/pubmed/23925405

Camacho Gómez, M. del S. (2011). Business stories in case studies. Pensamiento \& Gestión, (31), 196-210.

Castro Monge, E. (2010). El Estudio de Casos como Metodología de Investigación Científica en Dirección y Economía de la Empresa. Revista Nacional de Administración, 1(2), 31-54. doi: 10.1016/S11352523(12)60033-1

Chan, S., Inoue, C., yTaylor, L. (2015). Developing rubrics to assess the reading-into-writing skills: A case study. Assessing Writing, 26, 20-37. doi: 10.1016/j.asw.2015.07.004

Clary, R. M., Brzuszek, R. F., y Fulford, C. T. (2011). Measuring Creativity: A Case Study Probing Rubric Effectiveness for Evaluation of Project-Based Learning Solutions. Creative Education, 02(04), 333340. doi: $10.4236 / c e .2011 .24047$

Codina, L., y Morales, A. (2019). Mendeley: red social, descubrimiento de información y gestión de referencias. Recuperado de https://hdl.handle.net/10230/42281

Codina, LI. (2018). Sistemas de búsqueda y obtención de información: componentes y evolución. Anuario ThinkEPI, 12, 77. doi: 10.3145/thinkepi.2018.06

Codina, Lluís. (2017). Investigación con bases de datos. Estructura y funciones de las bases de datos académicas. Análisis de componentes y estudio de caso. Recuperado de https://hdl.handle.net/10230/28135

Corbetta, P., Fraile, C., y Fraile, M. (2007). Metodología y técnicas de investigación social. McGraw-Hill.

Crosbie, R. (2005). Learning the soft skills of leadership. Industrial and Commercial Training, 37(1), 45-51. doi: $10.1108 / 00197850510576484$

Culpin, V., Eichenberg, T., Hayward, I., y Abraham, P. (2014). Learning, intention to transfer and transfer in executive education. International Journal of Training and Development, 18(2), 132-147. doi: 10.1111/ijtd.12033

Culpin, V., y Scott, H. (2012). The effectiveness of a live case study approach: Increasing knowledge and understanding of "hard" versus "soft" skills in executive education. Management Learning, 43(5), 565-577. doi: 10.1177/1350507611431530 
Diefenbach, T. (2009). Are case studies more than sophisticated storytelling? Methodological problems of qualitative empirical research mainly based on semi-structured interviews. Quality \& Quantity, 43(6), 875-894. doi: 10.1007/s11135-008-9164-0

Dooley, L. M. (2002). Case Study Research and Theory Building. Advances in Developing Human Resources, 4(3), 335-354. doi: 10.1177/15222302004003007

Eisenhardt, K. M. (1991). Better Stories and Better Constructs: The Case for Rigor and Comparative Logic. Academy of Management Review, 16(3), 620-627. doi: 10.5465/amr.1991.4279496

Flyvbjerg, B., Crowe, S., Cresswell, K., Robertson, A., Huby, G., Avery, A., ... Yin, R. K. (2006). Case study as a research method. ERS-RUPRI Conference: Exploring Rural Entrepreneurship, 11(1), 370-388. doi: $10.1108 / 01409170210782990$

Gallardo, Y. (2013). Logical operators in scientific information research. Medwave, 13(09), e5820. doi: 10.5867/medwave.2013.09.5820

George, A. L. (2019). Case Studies and Theory Development: The Method of Structured, Focused Comparison (pp. 191-214). Springer. doi: 10.1007/978-3-319-90772-7_10

Gordillo, J., y Rodríguez, V. (2010). La rúbrica como instrumento pedagógico para la tutorización y evaluación de los aprendizajes en el foro online en educación superior. Pixel-Bit. Revista de Medios y Educación, 36, 141-149. Recuperado de http://www.sav.us.es/pixelbit/pixelbit/articulos/n36/11.pdf

Harland, T. (2014). Learning about case study methodology to research higher education. Higher Education Research $\{\&\}$ Development, 33(6), 1113-1122. Recuperado de http://dx.doi.org/10.1080/07294360.2014.911253\$5C\$nhttp://www.tandfonline.com/doi/full/10.10 80/07294360.2014.911253\$5C\$nhttp://www.tandfonline.com/doi/pdf/10.1080/07294360.2014.911 253

Keevy, M. (2016). Using case studies to transfer soft skills (also known as pervasive skills). Meditari Accountancy Research, 24(3), 458-474. doi: 10.1108/MEDAR-04-2015-0021

Llanes, J., y Massot, I. (2013). Evaluar a través de los estudios de casos. Metodología Del Caso. En Orientación, Capítulo $\quad 5 . \quad$ (52-65). Recuperado de http://diposit.ub.edu/dspace/bitstream/2445/52308/1/52210_Cap5.pdf

Maggin, D. M., Briesch, A. M., Chafouleas, S. M., Ferguson, T. D., y Clark, C. (2014). A Comparison of Rubrics for Identifying Empirically Supported Practices with Single-Case Research. Journal of Behavioral Education, 23(2), 287-311. doi: 10.1007/s10864-013-9187-z

Mahdi, O. R., Nassar, I. A., y Almuslamani, H. A. (2020). The Role of Using Case Studies Method in Improving Students' Critical Thinking Skills in Higher Education. International Journal of Higher Education, 9(2), 297. doi: 10.5430/ijhe.v9n2p297

Martínez Carazo, P. C. (2006). El método de estudio de caso: Estrategia metodológica de la investigación científica. Pensamiento y Gestión: Revista de La División de Ciencias Administrativas de La Universidad Del Norte, (20), 165-193. doi: 10.1055/s-0029-1217568

Morgan, S. J., Pullon, S. R. H., Macdonald, L. M., McKinlay, E. M., y Gray, B. V. (2016). Case Study Observational Research: A Framework for Conducting Case Study Research Where Observation Data Are the Focus. Qualitative Health Research, 27(7), 1060-1068. doi: 10.1177/1049732316649160

Naumes, W., y Naumes, M. (2015). The art \& craft of case writing. New York: Routledge.

Noblitt, L., Vance, D. E., y Smith, M. L. D. (2010). A comparison of case study and traditional teaching methods for improvement of oral communication and critical-thinking skills. Journal of College Science Teaching, 39(5), 26-32.

Ogliastri, E. (1998). El método de casos. Cali: Publicaciones Crea.

Rave, E., y Franco, J. G. (2011). Casos empresariales colombianos. Medellín: Ceipa. Recuperado de http://aplicaciones.ceipa.edu.co/biblioteca/biblio_digital/virtualteca/libros/ebook_casos_empresariales.pdf

Rendtorff, J. D. (2015). Case Studies, Ethics, Philosophy, and Liberal Learning for the Management Profession. Journal of Management Education, 39(1), 36-55. doi: 10.1177/1052562914562282 
Ridder, H.-G. (2017). The theory contribution of case study research designs. Business Research, 10(2), 281305. doi: $10.1007 / \mathrm{s} 40685-017-0045-z$

Riddle, E. J., Smith, M., y Frankforter, S. A. (2016). A Rubric for Evaluating Student Analyses of Business Cases. Journal of Management Education, 40(5), 595-618. doi: 10.1177/1052562916644283

Shivakumar, K. (2012). The Case Study Method in Training and Management Education. IUP Journal of Soft Skills, 6(2), 55-64. Recuperado de http://search.ebscohost.com/login.aspx?direct=true\&db=bth\&AN=97723209\&site=ehost-live

Shugan, S. (2006). Editorial: Save Research: - Abandon the Case Method of Teaching. Marketing Science, 25(2), 109-115. doi: /10.1287/mksc.1060.0202

Siggelkow, N. (2007). Persuasion with case studies. Academy of Management Journal, 50(1), 20-24.

Sridevi, K. B. (2012). Effectiveness of Case Study in Life Skill Development: An Analysis. IUP Journal of Soft Skills, VI(3), 48-55.

Stake, R. E. (1995). The art of case study research. USA: University of Illinois at Urbana-Champaign.

Stake, R. E. (2004). Case Studies. En Reinforcement Learning an Introduction (pp. 236-247). The Howorth Press. doi: 10.1300/Jo04V20n03_08

Tecnológico de Monterrey. (2010). Enseñar con el método de casos. Recuperado de https://bit.ly/2LIszBQ

Wai, C. C., y Seng, E. L. K. (2013). Measuring the effectiveness of blended learning environment: A case study in Malaysia. Education and Information Technologies, 1-15. doi: 10.1007/s10639-013-9293-5

Welch, C., Piekkari, R., Plakoyiannaki, E., y Paavilainen-Mäntymäki, E. (2011). Theorising from case studies: Towards a pluralist future for international business research. Journal of International Business Studies, 42(5), 740-762. doi: 10.1057/jibs.2010.55

Yacuzzi, E. (2005). El Estudio De Caso Como Metodología De Investigación: Teoría, Mecanismos Causales, Validación. Recuperado de https://ucema.edu.ar/publicaciones/download/documentos/296.pdf

Yin, R. K. (2012). Case study methods. En APA handbook of research methods in psychology, Vol 2: Research designs: Quantitative, qualitative, neuropsychological, and biological. (pp. 141-155). Washington: American Psychological Association. doi: 10.1037/13620-009

Yin, R. K. (2013). Applications of case study research. Applied Social Research Methods Series, 34, 173. doi: 10.1097/FCH.ob013e31822ddage 